\title{
The role of pre-conditioning frequency in the experimental characterisation of hyper-elastic materials as models for soft tissue applications
}

\author{
SERENA DE GELIDI, GIANLUCA TOZZI and ANDREA BUCCHI* \\ School of Engineering, University of Portsmouth \\ Anglesea road, Portsmouth, PO1 3DJ, United Kingdom
}

\begin{abstract}
Rubber-like materials as many soft tissues can be described as incompressible and hyperelastic materials. Their comparable elastic behaviour, up to a certain extent, has been exploited to develop and test experimental methodologies to be then applied to soft biological tissues such as aortic wall. Hence, theoretical and experimental simulation of aortic tissue, and more generally blood vessel tissue, has been often conducted using rubbers.

Despite all the efforts in characterising such materials, a clear and comprehensive testing procedure is still missing. In particular, the influence of pre-conditioning in the mechanical response of hyper-elastic materials has been often neglected. In this paper, the importance of pre-conditioning is demonstrated by: 1. exploring the effect of stretching frequency applied before the uniaxial tensile test; 2. recognizing the role of specimen geometry and strain amplitude; 3 . verifying the impact of experimental data acquisition on finite element predictions.

It was found that stress-strain relationship shows a statistical difference between some frequencies of pre-conditioning and its absence. Only certain pre-conditioning frequencies were able to generate repeatable experimental data for strip or dumb-bell shapes. This feature corresponds to a consistent reduction in the scatter of critical pressures obtained by numerical simulations.

Keywords: Hyper-elastic material; Uniaxial test; Pre-conditioning; Instability; Finite element analysis.
\end{abstract}

\section{Introduction}

Rubber-like materials exhibit an elastic behaviour in a wide range of strains. This feature is exploited in the biomechanical field to obtain a valuable model of main arteries, due to their hyper-elastic behaviour [Holzapfel, 2005]. The use of synthetic materials facilitates the setting up of novel experimental techniques aiming at a more precise and reliable characterisation of biological tissues. This is mainly due to minor constraints on storage and sample preparation as well as availability and

*email: andrea.bucchi@port.ac.uk 
consistency in their mechanical properties, which makes polymers perfect substrates to implement or refine various experimental techniques [Tozzi et al., 2013]. Moreover, rubber minimizes the effect of material variability that is quite significant in biological tissue.

Rubbers have been widely adopted to reproduce and study aortic disease, such as aneurysm. Schurink et al. [Schurink et al., 1998] created a rubber model of aneurysm to study the effect of endoleakage, frequently observed after endovascular repair of abdominal aortic aneurysm. Holzapfel et al. [Holzapfel et al., 2002; Holzapfel, 2005] showed that arteries share a thermomechanical behaviour similar to rubber-like materials. In order to clarify the effect of the intraluminal thrombus on the transmission of pressure, Hinnen et al. [Hinnen et al., 2005] modelled saccular aneurysms made of elastic vulcanized rubber. Aiming to identify aneurysm sites of rupture, Doyle et al. [Doyle et al., 2009b,a] implemented silicone experimental models. Similarly, Segers and Verdonck [Segers and Verdonck, 2000] replicated the human arterial tree to study the pressure wave propagation along the arteries.

Moreover, in order to carry out numerical predictions (i.e. calculating the critical pressure for aneurysm formation) reliable experimental mechanical properties need to be obtained.

Generally, hyper-elastic materials are mathematically described by means of strainenergy functions [Holzapfel, 2000]. The calibration of these functions is based on the fitting of experimental data obtained by uniaxial tensile test, equi-biaxial test and pure shear [Kawabata et al., 1981]. The uniaxial tensile test represents the preferred approach to obtain mechanical properties [Sokolis et al., 2002; Shergold et al., 2006; Vahapoglu et al., 2011], due to its simple setup and to its limited cost compared to other more complex tests, such as indentation or inflation.

Therefore, the testing methodology constitutes a critical aspect for a proper characterization of hyper-elastic materials. In literature, a number of studies reported the effect of actuator speed on the uniaxial tensile test. For example, Jerabek et al. [Jerabek et al., 2010] elongated polypropylene specimens at $0.01 \mathrm{~mm} / \mathrm{s}$, Cox et al. [Cox et al., 2008] stretched polydimethylsiloxane dumb-bells at $0.2 \mathrm{~mm} / \mathrm{s}$, ISO standard [ISO 37, 2005] suggests to test small dumb-bells at $3.33 \mathrm{~mm} / \mathrm{s}$, while Shergold et al. [Shergold et al., 2006] preferred to pull their silicone dumb-bells at 8.33 $\mathrm{mm} / \mathrm{s}$.

Another crucial aspect of the testing procedure is the pre-conditioning stage, during which rubber-like materials exhibit an evident change in their mechanical properties. After the first cycle of stretching and recovering the material softens, meaning that in the following cycles lower stresses are generated for the same applied strain. Carbon filled rubbers are particularly sensitive to this behaviour [Gent, 2001]. This phenomenon, referred as Mullins effect, proves that a mechanical behaviour is repeatable only after a number of pre-conditioning cycles [Diani et al., 2009]. Therefore, this aspect cannot be neglected in any experimental protocol.

In addition, there is no standard number of pre-conditioning cycles (often referred 
as scragging) to perform before uniaxial tensile test. As an example, no indication of pre-conditioning is given by ASTM [ASTM D 412 -06 a, 2009]. Doyle et al. [Doyle et al., 2009b] preconditioned silicone samples by 10 cycles, before carrying out the uniaxial tensile test. Differently, British Standard [BS 903-1, 1995] suggests to perform 5 cycles in order to improve test reproducibility. However, rarely the value of frequency selected to perform the pre-conditioning is reported [Sahakaro and Beraheng, 2008] and attempts in incorporating pre-conditioning guidelines into ISO standards have been problematic so far, as preliminary trials have failed to produce evidence that pre-conditioning effect is large enough to be significant [Brown, 2006]. Numerical methods can supply the missing evidences, assessing the effect of experimental data collection on finite element predictions, such as a tube inflation. It is well-known that cylindrical rubber membranes subjected to inflation experience large deformations [Kanner and Horgan, 2007]. Nonlinearities related to the material and the geometry interact to exhibit certain interesting instabilities and/or bifurcations [Gent, 2005]. The limit point (critical pressure) occurring during the inflation of long tubes is manifested by the appearance of a non-uniform deformation of the cross-section: one portion of the tube experiences the formation of a bulge, called aneurysm in medical context, while the remaining part is slightly inflated. Since 1891 this phenomenon has been widely described for rubbers [Mallock, 1890; Alexander, 1971; Kyriakides and Chang, 1990; Kyriakides and Yu-Chung, 1991; Kanner and Horgan, 2007]. More recently, Bucchi and Hearn [Bucchi and Hearn, 2013a,c] provided an exhaustive continuum mechanics background followed by numerical studies on distensible tubes. In order to describe how critical pressure may be predicted, five distinct elements with up to seven alternative strain-energy functions were evaluated in finite element analysis [Bucchi and Hearn, 2013c]. In contrast to previous works [Kyriacou and Humphrey, 1996; Holzapfel, 2005], most recent studies [Fu et al., 2012; Rodríguez and Merodio, 2011; Alhayani et al., 2013, 2014 ] suggest that the formation of arterial aneurysm can be regarded as an elastic instability problem.

In this paper, a finite element analysis is designed to assess the impact of the tensile stress-strain behaviour on associated numerical predictions. Thus, a cylindrical rubber tube, intended as a simplified aortic model, is inflated for the sake of comparing predicted values of critical pressure, associated to aneurysm formation, as function of the experimental data supplied. Such data refer exclusively to the response ob-served after the completion of pre-conditioning cycles. This is in agreement with the common recommendation to record stress-strain measurements only after preconditioning [Gent, 2001; 903-5, 2004; Brown, 2006]. Analogously, the exclusion of pre-conditioning cycles from subsequent analyses has been performed in recent studies [Dorfmann and Ogden, 2003; Hariharaputhiran and Saravanan, 2016]. Dorfmann and Ogden [Dorfmann and Ogden, 2003] presented a new pseudo-elastic constitu-tive model, specifying that the actual uniaxial response of the carbon filled rubber was taken only after six pre-conditioning cycles. The electrical resistivity-strain re- 
de Gelidi et al.

sponse of carbon filled rubbers was measured by De Focatiis et al. [De Focatiis et al., 2011] after four preliminary cycles. Hariharaputhiran and Saravanan [Hari-

haraputhiran and Saravanan, 2016] mentioned that only data collected from the last cycle were used to evaluate the stored energy of rubber sheets. In a seminal study for arterial behaviour, Fung et al. [Fung et al., 1979] indicated that strain-energy functions should be adopted after pre-conditioning, as the internal structure of the artery is rearranged during the first cycles. Hence, the material softening is not included in the mathematical formulation of the strain energy-functions in order to describe the hyper-elastic behaviour after the application of a pre-conditioning stage. In this study, the role of the pre-conditioning frequency $\left(f_{P}\right)$, adopted during the experimental testing, is shown to affect the predicted magnitude of the critical pressure. In other words, each $f_{P}$ leads to a distinct mechanical behaviour of the same material, thus numerical predictions are biased by such experimental feature. Several strain-energy functions are adopted to model such variety of experimental responses and to offer a detailed comparison of numerical outcomes.

The main aim of this work consists in showing, experimentally, the effect of the frequency adopted during the mechanical pre-conditioning on the uniaxial tensile outcomes. Furthermore, such effect is corroborated numerically, associating such frequencies, selected for experimental testing, to aneurysm predictions.

In addition, two further aspects are explored: the influence of the sample geometry on the stress-strain curves and the strain amplitude of the pre-conditioning cycles. Despite the fact that the uniaxial tensile test provides a brief characterization of material properties, the results reported in this study are sufficient to demonstrate the importance of the frequency value adopted during the mechanical pre-conditioning for a particular material and shape.

\section{Materials and methods}

\subsection{Materials and specimen}

Strips and dumb-bells are prepared out of a carbon filled rubber material produced by Avon Rubber. Aiming at designing an experimental protocol for aortic tissue, small specimens are required. Thus, the dumb-bell type 4 described in ISO 37:2005 (E) is adopted in this study as the smallest standard available [ISO 37, 2005]. Strips dimensions ( $35 \mathrm{~mm} \times 2 \mathrm{~mm}$ ), as analogous to the narrow part of the dumb-bell, are also tested. In both cases, a uniform thickness of $1.9 \mathrm{~mm}$ is calculated as average of optical confocal microscopy measurements on 10 samples. Dumb-bells are cut by means of a custom-made die (Gibitre Instruments, Italy). Differently, strips are handcut by means of a wet scalpel and carefully selected after calibre measurements, discarding samples with a tolerance of $1 \%$ in length and $10 \%$ in width. 


\subsection{Experimental procedures}

Samples are subjected to a pre-conditioning process as recommended in [BS 9031, 1995; ASTM D 1776 -04, 2004; Doyle et al., 2009a] to overcome the Mullins effect and stretched until rupture, immediately after such loading-unloading cycles. Therefore, any kind of relaxation effect is avoided [Gent, 2001].

\subsubsection{Pre-conditioning details}

The pre-conditioning stage is carried out using a sinusoidal load waveform, whose strain amplitude is 0.7 ; calculated as the $10 \%$ of the maximum strain $(7 \mathrm{~mm} / \mathrm{mm})$. Such ultimate value was obtained via preliminary uniaxial tests performed without pre-conditioning. Furthermore, as recommended by British Standard [BS 903-1, 1995], 5 cycles of pre-conditioning are performed.

\subsubsection{Experimental setup}

Specimens are tested uniaxially using a MTS Landmark (MTS Systems Corporation, USA) equipped with a $2.5 \mathrm{kN}$ load cell (Fig. 1). The sample is kept in position with BOSE grips Assy 3200 (Bose Corporation, USA). The displacement is measured using a Messphysik video-extensometer with a field of view ranging from $0.4 \mu \mathrm{m}$ to $50 \mathrm{~mm}$ (Messphysik Materials Testing GMBH, Austria). The recording is activated before any movement of the crosshead and stopped manually after the end of the test. Following the pre-conditioning stage, each sample is tested up to rupture under displacement control, at a constant cross-head speed of $0.2 \mathrm{~mm} / \mathrm{s}$. Such speed value was calculated as a representative (average) value of low speeds adopted in literature [ISO 37, 2005; Shergold et al., 2006; Cox et al., 2008; Jerabek et al., 2010].

[Fig. 1 about here.]

\subsubsection{Testing Methods}

Three major experimental analyses are carried out, to investigate and assess different aspects of the pre-conditioning.

- In order to determine the influence of the $f_{P}$ on the mechanical stress-strain response, a number of frequencies have been tested: $0.01 \mathrm{~Hz}, 0.05 \mathrm{~Hz}, 0.075 \mathrm{~Hz}$, $0.1 \mathrm{~Hz}, 0.2 \mathrm{~Hz}, 0.3 \mathrm{~Hz}, 0.4 \mathrm{~Hz}, 0.5 \mathrm{~Hz}, 0.6 \mathrm{~Hz}, 0.8 \mathrm{~Hz}, 1 \mathrm{~Hz}, 1.2 \mathrm{~Hz}, 1.4 \mathrm{~Hz}, 1.6$ $\mathrm{Hz}$ and $2 \mathrm{~Hz}$. Moreover, a test without pre-conditioning, intended as a control, is carried out. Four specimens $(n=4)$ are tested for each frequency.

- The effect of sample shape on the material characterization is also assessed. Rubber dumb-bells are tested at the following pre-conditioning frequencies: $0.2 \mathrm{~Hz}$, $0.6 \mathrm{~Hz}, 0.8 \mathrm{~Hz}, 1 \mathrm{~Hz}, 1.4 \mathrm{~Hz}$ and $2 \mathrm{~Hz}$. Similarly, a control test without any pre-conditioning is performed. 
de Gelidi et al.

- Subsequently, another aspect of the pre-conditioning process is explored: the strain magnitude. In two distinct tests, conducted at $0.2 \mathrm{~Hz}$, two strain amplitudes are investigated: $20 \%$ and $8.5 \%$ of the maximum strain recorded.

Despite the specific experimental analysis conducted, the post-processing stage is common to all samples. The engineering stress is calculated taking into account a constant undeformed cross-section area, assuming the thickness of all samples equal to the calculated average value of $1.9 \mathrm{~mm}$. In addition, such stress-strain data are filtered using a home-written Matlab (Mathworks, Massachusetts, US) script, which reduces the number of experimental data to $40 \%$ of the original dataset performing a block average. The effect of pre-conditioning frequencies on the tensile behaviour was evaluated by one-way ANOVA test.

\section{Finite element model}

In order to simulate the inflation of a cylindrical membrane, a circular tube is created using Abaqus (Dassault Systmes S.A., France), in accordance with a typical geometry extensively used in literature [Shi and Moita, 1996; Bucchi and Hearn, 2013c] to assess aneurysm formation: external radius $10 \mathrm{~mm}$, thickness $1 \mathrm{~mm}$ and length $200 \mathrm{~mm}$.

Four node shell elements with reduced integration (S4R) are adopted to mesh the designed tube with a radius equal to $9.5 \mathrm{~mm}$, representing the radius of the middle plane between the internal and external cylindrical surface [Lopes et al., 2007; Gonçalves et al., 2008].

A mesh sensitivity study is carried out to prove independence of results from the adopted discretization, and at the same time to optimize the computational cost. The coarsest mesh (Mesh 1) is formed by 8 elements along the circumference and 44 elements along the longitudinal direction, for a total number of 352 elements. Subsequent refinements are obtained doubling elements in both directions. Hence, Mesh 2 is constituted by 1408 elements, Mesh 3 by 5632 elements and the finest Mesh 4 by 22528 elements.

Both tube ends are fully constrained (suppressing displacements and rotations) and an internal inflating pressure with an initial magnitude of $1 \mathrm{kPa}$ is applied. Elastic instability is analysed by the modified Riks method [Wriggers, 2010], which provides a load proportional factor $(\mathrm{LPF})$ to be interpreted as a multiplier of the initial load (pressure) applied. Below the maximum LPF, the model is deforming under static equilibrium. The peak, instead, represents the critical pressure that causes the aneurysm formation, hence instability.

Material properties are defined by experimental responses observed immediately after the pre-conditioning. Such experimental data are fitted using strain-energy functions dependent on the first strain invariant such as Neo-Hookean [Treloar, 1943]

$$
W=C_{10}\left(I_{1}-3\right)
$$


and, intended as a generalisation of the previous, Yeoh [Yeoh, 1993] formulation

$$
W=C_{10}\left(I_{1}-3\right)+C_{20}\left(I_{1}-3\right)^{2}+C_{30}\left(I_{1}-3\right)^{3}
$$

where $C_{10}, C_{20}$ and $C_{30}$ are fitting coefficients. The eight chain model presented by Arruda and Boyce [Arruda and Boyce, 1993] adopts an invariant and a stretch:

$$
W=\mu \sum_{i=1}^{5} \frac{C_{i}}{\lambda_{m}^{2 i-2}}\left(I_{1}^{i}-3^{i}\right)
$$

where $\mu$ is a material parameter function of the initial shear modulus $\mu_{0}, C_{i}$ are related to the series expansion form of the inverse Langevin function and $\lambda_{m}$ is the locking stretch. Other strain energy functions are function of the first and second invariant implicitly, such as Ogden [Ogden, 1972]:

$$
W=\mu \sum_{i=1}^{N} \frac{\mu_{i}}{\alpha_{i}}\left(\lambda_{1}^{\alpha_{i}}+\lambda_{2}^{\alpha_{i}}+\lambda_{3}^{\alpha_{i}}-3\right)
$$

where $\mu_{i}$ and $\alpha_{i}$ are temperature-dependent material parameters that allow a good fit of the theoretical description to the experimental data [Ogden, 1972]. In Abaqus, the factor multiplying the sum of deviatoric principal stretches is different, being $2 \mu_{i} / \alpha_{i}^{2}$.

\section{Results}

\subsection{Experimental results}

Aiming to focus on the mechanical tensile response obtained immediately after the pre-conditioning process, the loading-unloading paths are not displayed. Therefore, the mechanical behaviour can be easily appreciated even for strain below 1 .

\subsubsection{Pre-conditioning of strips}

Strips have been pre-conditioned for 5 cycles at 7 different frequencies and stretched until rupture. In addition, a control test without pre-conditioning has been performed. As shown in Fig. 2, strips stress-strain responses appear to be heterogeneous. The range of maximum stress recorded is highly variable, in the range 1.46 $\mathrm{MPa}-5 \mathrm{MPa}$. For the particular material and geometry selected pre-conditionings of $0.2 \mathrm{~Hz}, 0.6 \mathrm{~Hz}$ and above $1.2 \mathrm{~Hz}$, generate a quite repeatable behaviour, reducing the scatter between the lowest and the highest maximum stress value to just $20 \%$. The strain values in each plot associated to $\sigma_{\text {avg }}$, which is calculated as half of the $\min \left\{\sigma_{1}^{\max }, \sigma_{2}^{\max }, \ldots, \sigma_{n}^{\max }\right\}$, were considered. In this case, the strain obtained in absence of pre-conditioning is significantly different $(p=0.005)$ in relation to preconditioning frequencies $f_{P} \geq 1 \mathrm{~Hz}$. Differently, no sensible variation $(p=0.634)$ in the curve stiffness evaluated for $\sigma_{\text {avg }}$ was observed.

[Fig. 2 about here.] 


\subsubsection{Pre-conditioning of dumb-bells}

Dumb-bells are tested in order to explore the effect of a geometric variation on experimental data collection, using the same experimental setup and methodology adopted for the strips.

In the vast majority of the stress-strain plots (Fig. 3) stress values appear scattered, except for some particular frequencies (i.e. $1 \mathrm{~Hz}$ ) where stress-strain curves are well overlapped. The ANOVA test on the stiffness, evaluated at $\sigma_{\text {avg }}$, revealed that the presence of pre-conditioning generates statistically different $(p=0.0022)$ material elastic response. Similarly to the strips, a statistical evidence $(p=1.17 * 10-7)$ is observed analysing the strain values associated to $\sigma_{\text {avg }}$.

[Fig. 3 about here.]

\subsubsection{Amplitude response}

Differently from the previous two experimental analyses, the strain magnitude of the cycles has been investigated: a comparison between $20 \%$ and $8.5 \%$ of the maximum strain recorded without pre-conditioning has been carried out.

Tensile test plots are quite comparable if the strain ratio is in the range $0-2$. Differently, for a strain ratio of 3 , results for pre-conditioning amplitude of $20 \%$ show a difference of 0.6 MPa between the maximum and the minimum stress calculated. This range is exactly reduced by $50 \%$ for a pre-conditioning amplitude of $8.5 \%$.

\subsection{Finite elements results}

Firstly, a mesh sensitivity study compared the predicted maximum load proportional factor (LPF), leading to formation of a bulge, for different strain-energy functions. As a result, Mesh 3 (5632 elements) is deemed to be an appropriate mesh, as the calculated LPF value just differs of $0.4 \%$ compared to the more computational demanding Mesh 4 . The latter mesh predicted the minimum critical pressure associated to the second order Ogden model $(21.65 \mathrm{kPa})$ and the maximum value $(28.16 \mathrm{kPa})$ with a Neo-Hookean.

The role of $f_{P}$ chosen during the experimental procedure is reflected on the numerical predictions for the critical pressure shown in Table 1 for the strips and Table 2 for the dumb-bells. The word unstable indicates that the curve fitting procedure in ABAQUS, declares the material to be unstable over the specified range of strain. Whether the material is stable but no critical pressure is predicted a symbol $(\bullet)$ is reported, meaning that the aneurysm is not formed.

[Table 1 about here.]

[Table 2 about here.] 
As an example of the fitting procedure, the softest stress-strain experimental response obtained after a pre-conditioning at $0.2 \mathrm{~Hz}$ is reported in Fig. 4. Such data-set is fitted by five different strain-energy functions: Arruda-Boyce, Neo-Hooke, Yeoh, Ogden $1^{\text {st }}$ order and Ogden $2^{\text {nd }}$ order (Table 3 ). Despite the stability computed by Abaqus in the range displayed, substantial differences can be appreciated between the models and their fitting capabilities. Neo-Hookean modelling appears stiffer compared to other strain-energy functions up to strain 1.5 and far softer than the others above such deformation. Differently, Yeoh and Ogden $2^{\text {nd }}$ order compute the response closest to the experimental data along the whole range of strain.

\section{[Table 3 about here.]}

[Fig. 4 about here.]

Strips results show that Neo-Hookean strain-energy function always predicts the highest limit of instability, while the Ogden models estimates the lowest values (Fig. $5 \mathrm{~A})$. The absence of pre-conditioning enlarges by far the range of critical pressures $(15.2 \mathrm{kPa}-28.3 \mathrm{kPa})$, which corresponds to a $\Delta P=P \max -P \min =13.1 \mathrm{kPa}$. The use of $0.2 \mathrm{~Hz}$ of pre-conditioning reduces this range $(20.2 \mathrm{kPa}-29.3 \mathrm{kPa})$, $\Delta P=9.1 \mathrm{kPa}$. Furthermore, this trend is enhanced for $0.6 \mathrm{~Hz}$ or $1.6 \mathrm{~Hz}$ that produce a pressure difference of $\Delta P=7.4 \mathrm{kPa}$ and $\Delta P=6.5 \mathrm{kPa}$, respectively.

[Fig. 5 about here.]

Dumb-bells simulations show similar trends observed in strips, as confirmed in Fig. 5B. The maximum pressure difference of $\Delta P=10.7 \mathrm{kPa}$ is observed in absence of pre-conditioning, in the range $17.4 \mathrm{kPa}-28.2 \mathrm{kPa}$. The $\Delta P$ is progressively reduced with the increasing of $f_{P}$, reaching the minimum difference $\Delta P=5.8 \mathrm{kPa}$ $(22.5 \mathrm{kPa}-28.2 \mathrm{kPa})$ at $1 \mathrm{~Hz}$ (Table 2$)$.

Despite different pressure differences are observed, the ANOVA tests deny any statistical evidence of the $f_{P}$ adopted to pre-cycle strips $(p=0.7)$ or dumb-bells $(p$ $=0.3)$.

[Fig. 6 about here.]

Fig. 6 illustrates how the selection of strain-energy functions affects the prediction of aneurysm formation in term of shape (deformations), associated radial strains and stress levels. Elastic tubes are represented just after the critical pressure is attained. In detail, model (A), (B) and (C) refer to the stiffest response obtained at $0.2 \mathrm{~Hz}$. It appears that the largest bulge is obtained for the Arruda-Boyce model (C).

Similarly, models (D), (E), and (F) belong to the softest behaviour at the same frequency (Fig. 6). The softest experimental response shows an analogous behaviour: 
the largest bulge is obtained with the Arruda-Boyce model (F).

Further ANOVA tests are performed on the maximum displacement instigated immediately after the formation of the bulge. No statistical relevance is reported for the experimental data collected from strips $(p=0.62)$. However, the displacements computed in the model based on dumb-bells, pre-conditioned at $f_{P}=1 \mathrm{~Hz}$, are statistically different $(p=0.01)$ from $f_{P}=0.2 \mathrm{~Hz}$ and from the control test.

\section{Discussions}

The main aim of this study is to justify the inclusion of some guidelines concerning the pre-conditioning stage in mechanical testing of hyper-elastic materials. Despite few attempts have been made to specify some aspects related to the adopted experimental protocol [Doyle et al., 2009a; Bailly et al., 2014], no indication concerning the $f_{P}$ has been encountered. The purpose of finite element analysis consists in quantifying, in terms of critical pressure values, the effect of distinct tensile behaviour exhibited after the pre-conditioning conducted at certain frequencies. Thus, such analysis represents a powerful tool to assess whether the experimental protocol may or not affect associated numerical predictions: differences in material properties may discriminate the predictions of aneurysm formation.

The results presented in this work seem to show, for the first time to the authors knowledge, that the effect of $f_{P}$ on $\mathrm{FE}$ results is quite significant.

Strips results (Fig. 2) show that, for the adopted material and shape, only selected frequencies produce well overlapped stress-strain curves: $0.2 \mathrm{~Hz}, 0.6 \mathrm{~Hz}, 1.2 \mathrm{~Hz}$ and $1.6 \mathrm{~Hz}$. However, only the highest of the investigated frequencies $(1.6 \mathrm{~Hz})$ generates a numerical prediction with the minimum difference in the critical pressure values, as clearly reported in (Table 1). Meier et al. [Meier et al., 2003] reported a limited scatter of stress-stretch responses obtained for silicone rubber strips. However, as the experimental protocol is not specified and being the material different, no comparison is feasible.

Dumb-bells stress-strain curves appear well overlapped when $f_{P}$ is $1 \mathrm{~Hz}$ (Fig. 3). In this case, experimental data are fitted by a larger number of strain-energy functions compared to other frequencies of pre-conditioning (Table 2). This $f_{P}=1 \mathrm{~Hz}$ produces the minimum difference in the predicted values of critical pressure for both strips and dumb-bells. Furthermore, ANOVA tests add a statistical evidence of the enlargement of the bulge obtained (Fig. 6).

Additionally, it has been observed that the largest bulge in not instigated by the highest critical pressure (Fig. 6). This aspect will be further investigated in future works.

In both sample shapes (strip and dumb-bell), the absence of pre-conditioning systematically generates less reproducible results, with a large range of stresses for the same interval of strains (Figs. 2 and 3). This effect is also reflected in the FE results, where the maximum scatter of critical pressures is achieved when samples are not preconditioned (Table 1 and 2). 
As additional remark, it appears clear the role of the strain-energy function fitting the experimental data on FE results. Comparing the plot in Fig. 4 and numerical results in Table 1 for the softest response at $0.2 \mathrm{~Hz}$, it seems that the highest critical pressure is associated to the stiffest stress-strain fitting below strain 1 , given by the Neo-Hookean model. However, it is worth notice that such mathematical model provides the poorest fitting of the experimental data-set, compared to the others. Therefore, further to the effect of the $f_{P}$ on the experimental mechanical response, the magnitude of numerical results appears affected by the selection of model fitting the stress-strain response. Despite Steinman et al [Steinmann et al., 2012] assessed fourteen constitutive models, they did not run a finite element analysis to quan-tify the effect of strain-energy functions on numerical outcomes. In addition, the constitutive sensitivity analysis carried out in the present work answers a key is-sue recently pointed out in literature: comparison between strain-energy functions requires that constitutive models are calibrated on the same experimental data set [Bucchi and Hearn, 2013b,c; Rodrı'guez-Martı'nez et al., 2015]. Although an opportune strain-energy function is an essential prerequisite for valuable numerical predictions [Marckmann and Verron, 2006], a constitutive model is generally preferred to others available for no clear reason [Shi and Moita, 1996; Scotti et al., 2008; Zhao et al., 2008; Ahn and Kim, 2010; Cloonan et al., 2012; Gokgol et al., 2012]. Shi and Moita [Shi and Moita, 1996] selected the Ogden model to investigate numerically the inflation of hyper-elastic membranes. Cloonan et al. [Cloonan et al., 2012] preferred a Mooney-Rivlin strain-energy function [Mooney, 1940] to model the indentation of silicone membranes. The freedom in selecting a constitutive model is even more evident for hyper-elastic tissues. An Ogden-type of strain-energy function has been adopted by Zhao et al. [Zhao et al., 2008] to study the tension, torsion and bending of the aortic wall. Differently, Scotti et al. [Scotti et al., 2008] preferred the Mooney-Rivlin strain-energy function to model the aortic wall in a fluid-structure interaction analysis. In order to model soft tissue indentation, the liver has been described by a Neo-Hookean model [Ahn and Kim, 2010] and by a Mooney-Rivlin strain-energy function [Gokgol et al., 2012].

No information about the viscoelastic behaviour is reported, since it is beyond this paper aims and for the following reasons. Viscoelastic properties of carbon black filled rubber characterize the mechanical behaviour at relatively small strains, up to 0.01 [White and De, 2001]. The focus of this research is on tensile stress-strain measurement at strains larger than 1 . Furthermore, viscoelasticity is usually modelled after relaxation tests, since it governs rate-dependent effects [Amin et al., 2002; Marvalova, 2007]. However, in this study no time was allocated to relaxation during the experimental tests. Recently, Bódai and Goda [Bódai and Goda, 2011] proposed to identify large-strain viscoelastic parameters of generalized Maxwell-model from two tensile tests at different speeds. In the present investigation, no rate-dependent effects have been analysed, since the same speed $(0.2 \mathrm{~mm} / \mathrm{s})$ has been adopted in all tests. Thus, viscoelasticity is not considered to play a main role in the performed 
experiments and hence is not taken into account in the numerical model.

The explanation of how $f_{P}$ might affect the mechanical characterization of the material remains unclear. Hence, this requires further investigations to a microscopic level that are beyond the scope of this work. However, according to the Brownian motion theory, natural frequency generates an unique behaviour of molecules [Gent, 2001]. Alternatively, in cluster size studies of pre-conditioned samples, a particular frequency distinguishes rigid from fragile behaviour [Bhowmick, 2008]. It is also worth considering an eventual role of the Payne effect, which is a typical feature of filled rubbers. Such effect can be attributed to changes in the material microstructure (e.g. to breakage and reforming of weak physical bonds between the filler aggregates) as a result of the overall deformation [Luo et al., 2010]. This phenomenon is easily appreciable in a dynamic mechanical analysis (DMA), since it reveals a decrease in storage modulus and a maximum in loss modulus. Furthermore, it has recently been showed that the Payne effect depends on both the frequency and the amplitude of the excitation [H“"ofer and Lion, 2009; Luo et al., 2010]. Thus, such phenomenon could play a role in generating the peculiar macroscopic behaviour observed in the present study only at certain frequencies.

As limitation of this work, the rubber material is intended as an experimental model that shares aortic hyper-elastic behaviour, not its exact biological response.

\section{Conclusions}

The reported experimental and numerical analyses suggest the importance of investigating the frequency of pre-conditioning for data set acquisition in the experimental characterisation of selected hyper-elastic materials. The effect of pre-conditioning stage on a rubber material has been examined. As a result, some frequencies produce stress-strain responses statistically different from not pre-stretched specimens. Focusing on the pre-conditioning step, three main aspects have been experimentally investigated: the frequency, the geometry of the samples and the strain amplitude of the cycles.

The impact of several experimental data sets has been quantified numerically with the estimation of critical pressures responsible for aneurysm formation in an inflated cylindrical tube. Critical pressure values are affected by the strain-energy function used to fit experimental data. It has been proved that a good overlap of the experimental curves corresponds to a minimum difference in the critical pressures calculations and a broader selection in the fitting strain energy functions. Hence, a scatter in the acquisition of experimental data has a direct effect in the reliability of numerical predictions.

Therefore, as a conclusion of this work, authors recommend to apply a preconditioning to the material tested. Furthermore, it is also suggested to check a reasonable number of $f_{P}$ and, by any means, to clearly state which frequency is used for the selected material. Finally, the adoption of standard sample geometry 
(e.g. dumb-bells) would facilitate the comparison between results available in the state of art.

As additional outcome, the present work represents also a preliminary benchmark for future studies on aneurysm formation in arterial designs. It is indeed recognized that elastic instabilities arising during inflation are of considerable interest in the context of biological tissue, which exhibits a hyper-elastic behaviour. Future extension of this work will apply the current experimental and computational protocol to biological materials for the sake of investigating the aneurysm formation in the main arteries.

\section{Acknowledgements}

The authors gratefully acknowledge Mr Colin Lupton for his support during the experimental testing, and Mr Jan Kamenik who helped with some of the tests.

\section{References}

903-5, B. [2004] "Physical testing of rubber Part 5: Guide to the application of rubber testing to finite element analysis."

Ahn, B. and Kim, J. [2010] "Measurement and characterization of soft tissue behavior with surface deformation and force response under large deformations." Medical image analysis 14(2), 138-48.

Alexander, H. [1971] "Tensile instability of initially spherical balloons." International Journal of Engineering Science 9(1), 151-160.

Alhayani, A. A., Giraldo, J. A., Rodríguez, J. F. and Merodio, J. [2013] "Computational modelling of bulging of inflated cylindrical shells applicable to aneurysm formation and propagation in arterial wall tissue." Finite Elements in Analysis and Design 73, 20-29.

Alhayani, A. A., Rodríguez, J. F. and Merodio, J. [2014] "Competition between radial expansion and axial propagation in bulging of inflated cylinders with application to aneurysms propagation in arterial wall tissue." International Journal of Engineering Science 85, 74-89.

Amin, A. F. M. S., Alam, M. S. and Okui, Y. [2002] "An improved hyperelasticity relation in modeling viscoelasticity response of natural and high damping rubbers in compression: experiments, parameter identification and numerical verification." Mechanics of Materials 34, 75-95.

Arruda, E. M. and Boyce, M. C. [1993] "A three-dimensional constitutive model for the large stretch behavior of rubber elastic materials." Journal of the Mechanics and Physics of Solids 41(2), 389-412.

ASTM D 1776 -04 [2004] "Standard Practice for Conditioning and Testing Textiles." ASTM D 412 -06 a [2009] "Standard Test Methods for Vulcanized Rubber and Thermoplastic Elastomers. 
Bailly, L., Deplano, V., Lemercier, a., Boiron, O. and Meyer, C. [2014] "New experimental protocols for tensile testing of abdominal aortic analogues." Medical Engineering and Physics 36(6), 800-804.

Bhowmick, A. K., Current topics in elastomeric research (CRC Press, Boca Raton, 2008).

Bódai, G. and Goda, T. [2011] "A new, tensile test-based parameter identification method for large-strain generalized maxwell-model." Acta Polytechnica Hungarica 8(5), 89-108.

Brown, R., Physical testing of rubber. 4th ed. (Springer, New York, 2006).

BS 903-1 [1995] "Physical testing of rubber. Part 1: Guide to the selection and use of methods of test for rubber." .

Bucchi, A. and Hearn, G. E. [2013a] "Delay or removal of aneurysm formation in the Anaconda wave energy extraction device." Renewable Energy 55, 104-119.

Bucchi, A. and Hearn, G. E. [2013b] "Predictions of aneurysm formation in distensible tubes: Part A Theoretical background to alternative approaches." International Journal of Mechanical Sciences 71, 1-20.

Bucchi, A. and Hearn, G. E. [2013c] "Predictions of aneurysm formation in distensible tubes: Part B Application and comparison of alternative approaches." International Journal of Mechanical Sciences 70, 155-170.

Cloonan, A. J., O'Donnell, M. R., Lee, W. T., Walsh, M. T., De Barra, E. and McGloughlin, T. M. [2012] "Spherical indentation of free-standing acellular extracellular matrix membranes." Acta biomaterialia 8(1), 262-73.

Cox, M. A. J., Driessen, N. J. B., Boerboom, R. A., Bouten, C. V. C. and Baaijens, F. P. T. [2008] "Mechanical characterization of anisotropic planar biological soft tissues using finite indentation: experimental feasibility." Journal of biomechanics $\mathbf{4 1}(2), 422-9$.

De Focatiis, D. S. A., Hull, D. and Sanchez-Valencia, A., "Electromechanical hysteresis in filled elastomers." In S. Jerrams and N. Murphy, eds., "Constitutive models for rubber VII," (CRC Press, London, 2011), 241-246.

Diani, J., Fayolle, B. and Gilormini, P. [2009] "A review on the Mullins effect." European Polymer Journal 45(3), 601-612.

Dorfmann, A. and Ogden, R. W. [2003] "A pseudo-elastic model for loading, partial unloading and reloading of particle-reinforced rubber." International Journal of Solids and Structures 40(11), 2699-2714.

Doyle, B. J., Corbett, T. J., Callanan, A., Walsh, M. T., Vorp, D. A. and McGloughlin, T. M. [2009a] "An experimental and numerical comparison of the rupture locations of an abdominal aortic aneurysm." Journal of endovascular therapy: an official journal of the International Society of Endovascular Specialists 16(3), $322-35$.

Doyle, B. J., Corbett, T. J., Cloonan, A. J., O'Donnell, M. R., Walsh, M. T., Vorp, D. A. and McGloughlin, T. M. [2009b] "Experimental modelling of aortic aneurysms: novel applications of silicone rubbers." Medical engineering \&5 physics 
31(8), 1002-12.

Fu, Y. B., Rogerson, G. and Zhang, Y. [2012] "Initiation of aneurysms as a mechanical bifurcation phenomenon." International Journal of Non-Linear Mechanics $47(2), 179-184$.

Fung, Y.-C., Fronek, K. and Patitucci, P. [1979] "Pseudoelasticity of arteries and the choice of its mathematical expression Pseudoelasticity of arteries and the choice of its mathematical expression of Applied Mechanics." The American Physiological Society 237, H620-H631.

Gent, A. N., Engineering with Rubber: How to Design Rubber Components. 2nd ed. (Hanser, Munich, 2001).

Gent, A. N. [2005] "Elastic instabilities in rubber." International Journal of NonLinear Mechanics 40(2-3), 165-175.

Gokgol, C., Basdogan, C. and Canadinc, D. [2012] "Estimation of fracture toughness of liver tissue: experiments and validation." Medical engineering $\&$ physics $\mathbf{3 4}(7)$, $882-91$.

Gonçalves, P., Pamplona, D. and Lopes, S. [2008] "Finite deformations of an initially stressed cylindrical shell under internal pressure." International Journal of Mechanical Sciences 50(1), 92-103.

Hariharaputhiran, H. and Saravanan, U. [2016] "A new set of biaxial and uniaxial experiments on vulcanized rubber and attempts at modeling it using classical hyperelastic models." Mechanics of Materials 92, 211-222.

Hinnen, J.-W., Koning, O. H. J., Visser, M. J. T. and Van Bockel, H. J. [2005] "Effect of intraluminal thrombus on pressure transmission in the abdominal aortic aneurysm." Journal of vascular surgery 42(6), 1176-1182.

Höfer, P. and Lion, A. [2009] "Modelling of frequency- and amplitude-dependent material properties of filler-reinforced rubber." Journal of the Mechanics and Physics of Solids 57(3), 500-520.

Holzapfel, G. A., Nonlinear solid mechanics. A continuum approach for engineering. 1 ed. (John Wiley \& Sons Ltd, Chichester (UK), 2000).

Holzapfel, G. A., "Similarities between soft biological tissues and rubberlike materials." In P. E. Austrell and L. Keri, eds., "ConstitutiveModels for Rubber IV," (Balkema, Leiden, 2005), 607-617.

Holzapfel, G. A., Gasser, C. T. and Stadler, M. [2002] "A structural model for the viscoelastic behavior of arterial walls: Continuum formulation and finite element analysis." European Journal of Mechanics - A/Solids 21(3), 441-463.

ISO 37 [2005] "Rubber, vulcanized or thermoplastic. Determination of tensile stressstrain properties."

Jerabek, M., Major, Z. and Lang, R. [2010] "Strain determination of polymeric materials using digital image correlation." Polymer Testing 29(3), 407-416.

Kanner, L. M. and Horgan, C. O. [2007] "Elastic instabilities for strain-stiffening rubber-like spherical and cylindrical thin shells under inflation." International Journal of Non-Linear Mechanics 42(2), 204-215. 
Kawabata, S., Matsuda, M., Tei, K. and Hawai, H. [1981] "Experimental Survey of the Strain Energy Density Function of Isoprene Rubber Vulcanizate." Macromolecules 14, 154-162.

Kyriacou, S. and Humphrey, J. D. [1996] "Influence of size, shape and properties on the mechanics of axisymmetric saccular aneurysms." Journal of Biomechanics 29(8), 1015-1022.

Kyriakides, S. and Chang, Y. [1990] "On the inflation of a long elastic tube in the presence of axial load." International journal of solids structures $\mathbf{2 6}(9 / 10)$, 975-991.

Kyriakides, S. and Yu-Chung, C. [1991] "The initiation and propagation of a localized instability in an inflated elastic tube." International Journal of Solids and Structures 27(9), 1085-1111.

Lopes, S., Gonçalves, P. and Pamplona, D. [2007] "Influence of initial geometric imperfections on the stability of thick cylindrical shells under internal pressure." Communications in numerical methods in engineering 23, 577-597.

Luo, W., Hu, X., Wang, C. and Li, Q. [2010] "Frequency- and strain-amplitudedependent dynamical mechanical properties and hysteresis loss of CB-filled vulcanized natural rubber." International Journal of Mechanical Sciences 52(2), $168-174$.

Mallock, A. [1890] "Note on the Instability of India-rubber Tubes and Balloons when distended by Fluid Pressure." Proceedings of the Royal Society A: Mathematical, Physical and Engineering Sciences 49, 458-463.

Marckmann, G. and Verron, E. [2006] "Comparison of Hyperelastic Models for Rubber-Like Materials." Rubber Chemistry and Technology 79(5), 835-858.

Marvalova, B. [2007] "Viscoelastic properties of filled rubber. Experimental observations and Material Modelling." Engineering Mechanics 14(1), 81-89.

Meier, P., Khader, S., Preuß, R., Dietrich, J. and Voges, D., "Uniaxial and equibiaxial tension tests of silicone elastomer." In J. Busfield and A. Muhr, eds., "Constitutive Models for Rubber III," (Swets \& Zeitlinger, Lisse, 2003), 99-106.

Mooney, M. [1940] "A Theory of Large Elastic Deformation." Journal of Applied Physics 11(9), 582.

Ogden, R. W. [1972] "Large deformation isotropic elasticity - on the correlation of theory and experiment for incompressible rubberlike solids." Royal society 326(1567), 565-584.

Rodríguez, J. F. and Merodio, J. [2011] "A new derivation of the bifurcation conditions of inflated cylindrical membranes of elastic material under axial loading. Application to aneurysm formation." Mechanics Research Communications 38(3), 203-210.

Rodríguez-Martínez, J., Fernández-Sáez, J. and Zaera, R. [2015] "The role of constitutive relation in the stability of hyper-elastic spherical membranes subjected to dynamic inflation." International Journal of Engineering Science 93, 31-45.

Sahakaro, K. and Beraheng, S. [2008] "Reinforcement of maleated natural rubber 
by precipitated silica." Journal of Applied Polymer Science 109(6), 3839-3848.

Schurink, G., M. Aarts, N., Wilde, J., van Baalen, J., Chuter, T., Kool, L. and van Bockel, J. [1998] "Endoleakage after stent-graft treatment of abdominal aneurysm: Implications on pressure and imagingan in vitro study." Journal of Vascular Surgery 28(2), 234-241.

Scotti, C. M., Jimenez, J., Muluk, S. C. and Finol, E. A. [2008] "Wall stress and flow dynamics in abdominal aortic aneurysms: finite element analysis vs. fluidstructure interaction." Computer methods in biomechanics and biomedical engineering 11(3), 301-322.

Segers, P. and Verdonck, P. [2000] "Role of tapering in aortic wave reflection: hydraulic and mathematical model study." Journal of Biomechanics 33(3), 299306.

Shergold, O. A., Fleck, N. A. and Radford, D. [2006] "The uniaxial stress versus strain response of pig skin and silicone rubber at low and high strain rates." International Journal of Impact Engineering 32(9), 1384-1402.

Shi, J. and Moita, G. F. [1996] "The post-critical analysis of axisymmetric hyperelastic membranes by the finite element method." Computer Methods in Applied Mechanics and Engineering 7825(96), 265-281.

Sokolis, D. P., Boudoulas, H. and Karayannacos, P. E. [2002] "Assessment of the aortic stress-strain relation in uniaxial tension." Journal of biomechanics 35(9), 1213-1223.

Steinmann, P., Hossain, M. and Possart, G. [2012] "Hyperelastic models for rubberlike materials: Consistent tangent operators and suitability for Treloar's data." Archive of Applied Mechanics 82(9), 1183-1217.

Tozzi, G., Zhang, Q.-H., Tong, J., Guillen, T., Ohrndorf, A. and Christ, H.-J. [2013] "Mechanical characterisation of a metallic foam-cement composite under selected loading conditions." Journal of Materials Science: Materials in Medicine 24(11), 2509-2518.

Treloar, L. R. G. [1943] "Stress strain data for vulcanised rubber under various types of deformation." Transactions of the Faraday Society 40, 59-70.

Vahapoglu, V., Karadeniz, S. and Yazici, I. [2011] "Uniaxial Tensile Testing of Rubber-Like Materials." Experimental Techniques 35(1), 17-23.

White, J. R. and De, S. K., eds., Rubber Technologist's Handbook (Rapra Technology Limited, Shawbury (UK), 2001).

Wriggers, P., Nonlinear Finite Element Methods (Springer-Verlag, Berlin, 2010).

Yeoh, O. H. [1993] "Some forms of the strain energy function for rubber." Rubber Chemistry and Technology 66, 754-771.

Zhao, A. R., Field, M. L., Digges, K. and Richens, D. [2008] "Blunt trauma and acute aortic syndrome: a three-layer finite-element model of the aortic wall." European Journal of Cardio-thoracic Surgery 34(3), 623-629. 

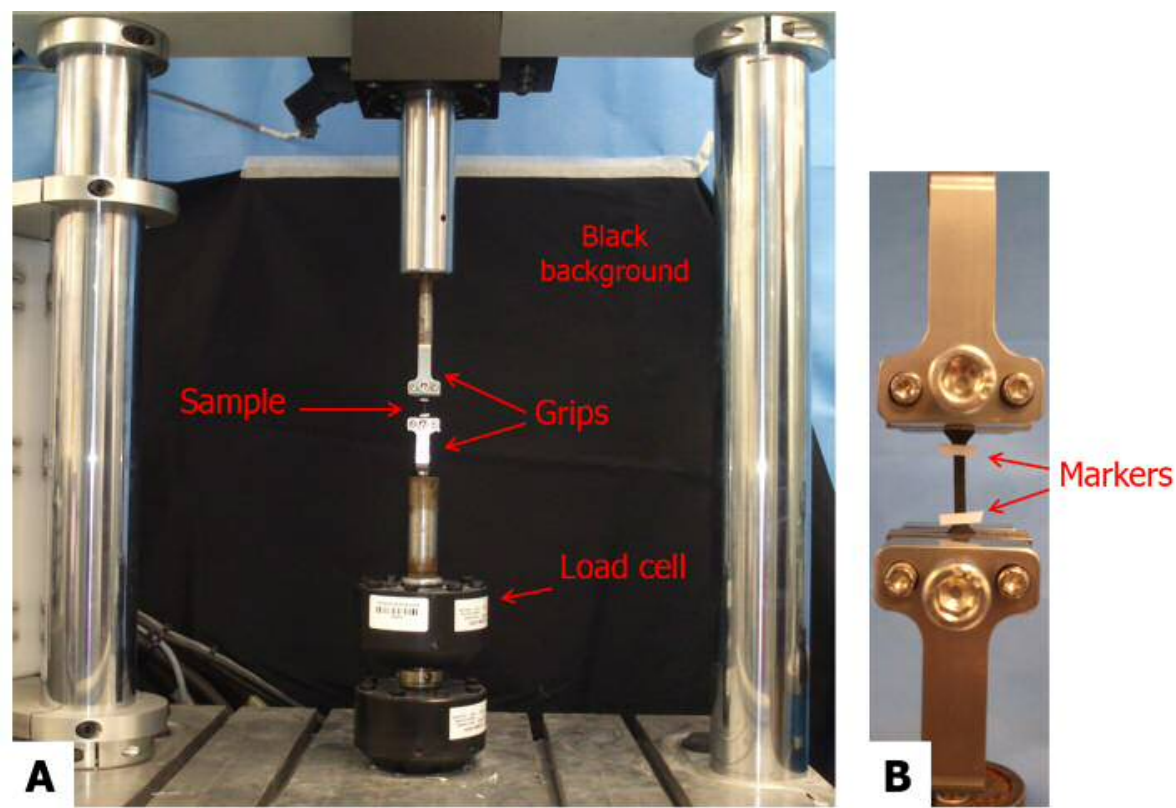

Fig. 1. A) Experimental setup adopted for the uniaxial tensile tests. The sample was kept in position by means of grips (B) and tested uniaxially up to failure. A video-extensometer was used to measure the displacement between the two white markers attached to the sample as detailed in $\mathrm{B}$. 



Fig. 2. Filtered uniaxial stress-strain response of rubber strips, pre-conditioned with 5 cycles at selected frequencies. Data obtained from no pre-conditioning, $0.2 \mathrm{~Hz}, 0.6 \mathrm{~Hz}$ and $1.6 \mathrm{~Hz}$ have been used in the FE model. 

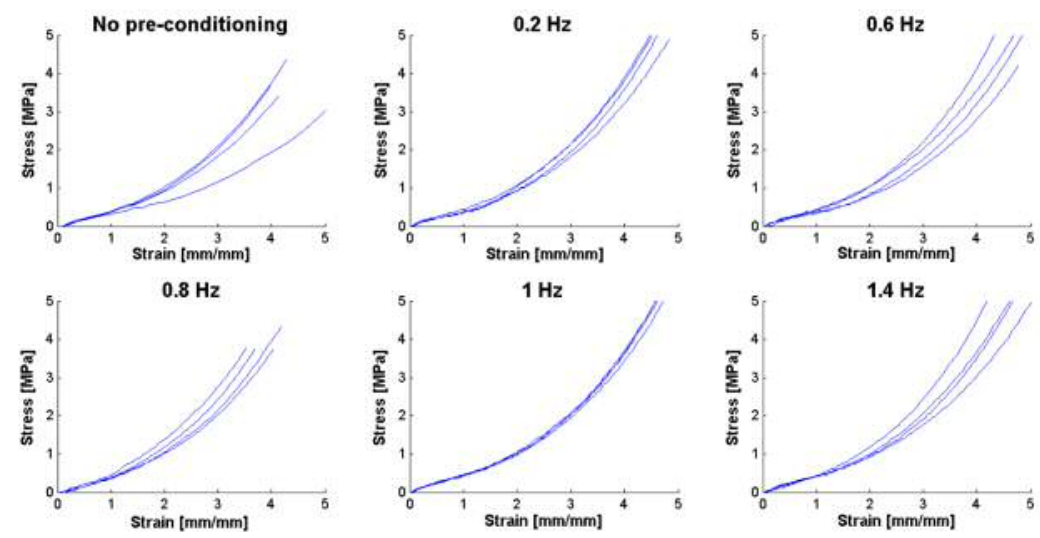

Fig. 3. Filtered uniaxial stress-strain response of rubber dumb-bells, pre-conditioned with 5 cycles at selected frequencies. Data obtained from no pre-conditioning, $0.2 \mathrm{~Hz}, 0.8 \mathrm{~Hz}$ and $1 \mathrm{~Hz}$ have been used in the FE model. 


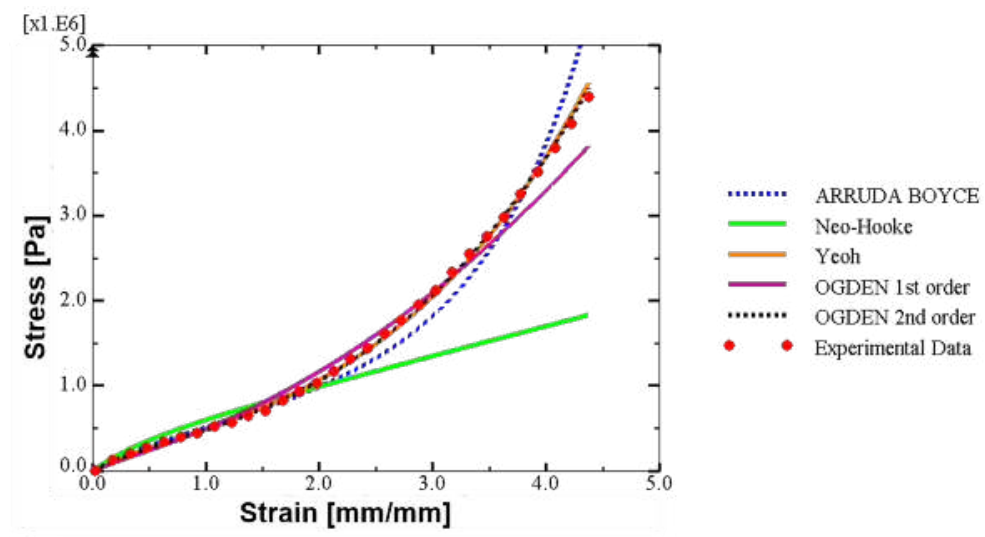

Fig. 4. Experimental data (red dots), collected from a strip pre-conditioned at $0.2 \mathrm{~Hz}$, fitted by different strain-energy functions in Abaqus. Fitting parameters are reported in Table 3. 

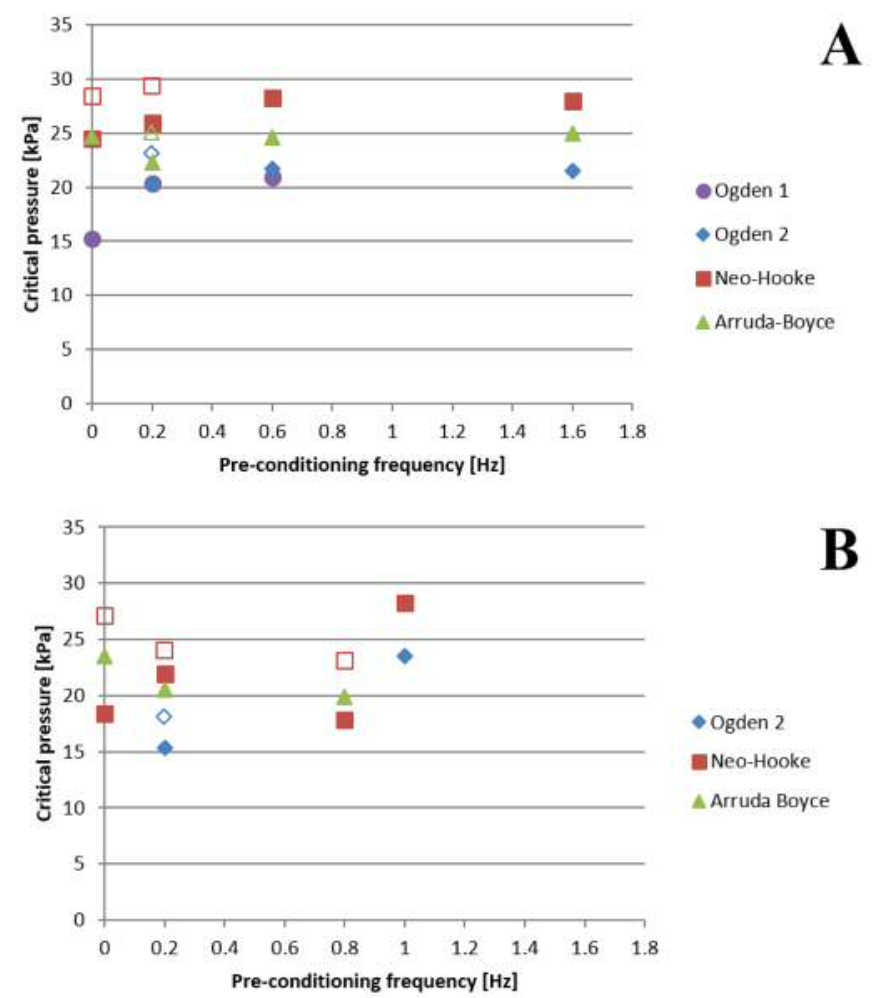

Fig. 5. Predicted critical pressures as a function of pre-conditioning frequencies: A) strips; B) dumb-bells. When two stress-strain curves, intended as the stiffest and the softest, have been selected, the higher values of critical pressure are indicated by open markers. 

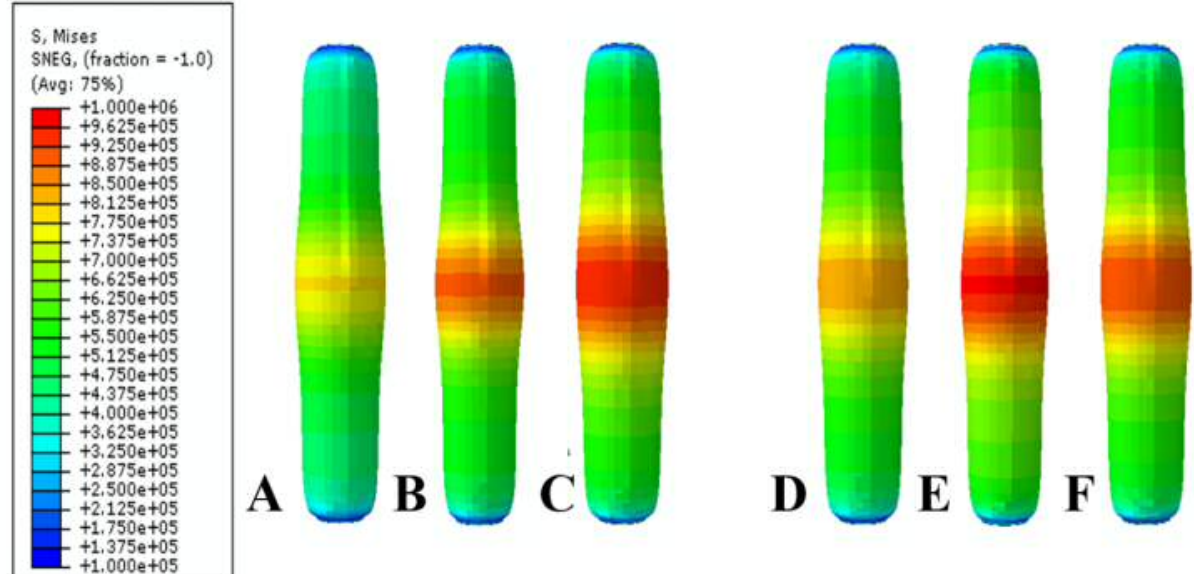

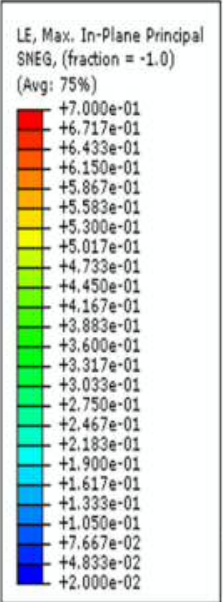
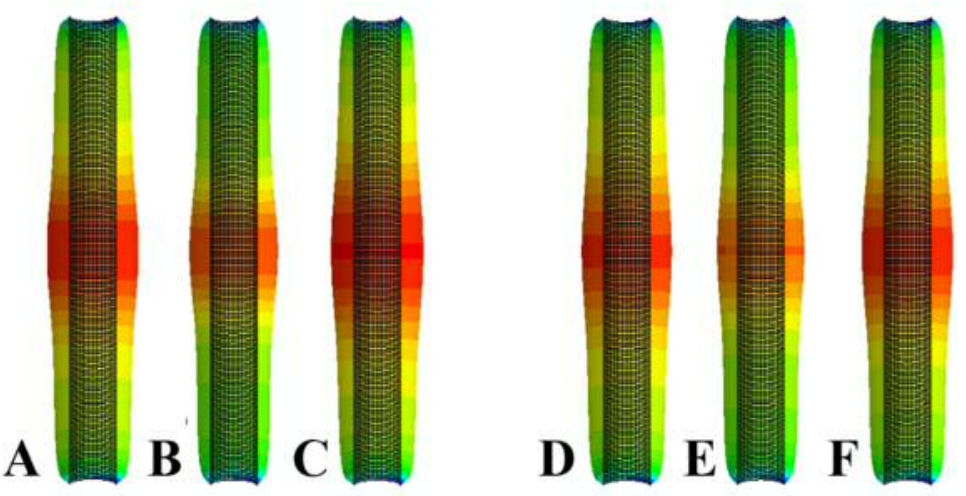

Fig. 6. Comparison of different aneurysm shapes predicted one step after the pressure reaches a critical point. The numerical results refers to experimental data for strips pre-conditioned at $0.2 \mathrm{~Hz}$, selecting two different stress-strain curves: the stiffest and the softest. The stiffest stressstrain response used: A) Ogden second order, B) Neo-hooke, C) Arruda-Boyce. The softest stressstrain response used: D) Ogden second order, E) Neo-hooke, F) Arruda-Boyce. The first row of results reports von Mises stress, whereas the second row shows the logarithmic strain valupes superimposed to the undeformed configuration. 
Table 1. Critical pressure values $[\mathrm{kPa}]$ calculated with the specified strain energy functions. The experimental data were obtained after strip pre-conditioning performed at the showed frequencies. Unstable indicates that no fitting is achieved and $\bullet$ specifies that no aneurysm was predicted. For $0 \mathrm{~Hz}$ and $0.2 \mathrm{~Hz}$ the stiffest (first column) and softest (second column) stress-strain response were taken into account. Conversely, an average curve was chosen for $0.6 \mathrm{~Hz}$ and $1.6 \mathrm{~Hz}$.

\begin{tabular}{|c|c|c|c|c|c|c|}
\hline \multirow{3}{*}{$\begin{array}{c}\text { Strain } \\
\text { energy } \\
\text { function }\end{array}$} & \multicolumn{6}{|c|}{ Pre-conditioning frequency } \\
\hline & \multicolumn{2}{|c|}{$0 \mathrm{~Hz}$} & \multicolumn{2}{|c|}{$0.2 \mathrm{~Hz}$} & \multirow[t]{2}{*}{$0.6 \mathrm{~Hz}$} & \multirow[t]{2}{*}{$1.6 \mathrm{~Hz}$} \\
\hline & Stiffest & Softest & Stiffest & Softest & & \\
\hline \multicolumn{7}{|l|}{ Ogden } \\
\hline $1^{s t}$ order & 15.275 & $\bullet$ & $\bullet$ & 20.316 & 20.892 & $\bullet$ \\
\hline Ogden & & & & & & \\
\hline $2^{\text {nd }}$ order & unstable & $\bullet$ & 20.215 & 23.111 & 21.734 & 21.489 \\
\hline Ogden & & & & & & \\
\hline $3^{r d}$ order & unstable & - & unstable & unstable & unstable & unstable \\
\hline Neo-Hooke & 24.447 & 28.392 & 25.931 & 29.327 & 28.290 & 27.951 \\
\hline Arruda-Boyce & unstable & 24.640 & 22.233 & 25.014 & 24.567 & 25.009 \\
\hline Yeoh & 17.358 & $\bullet$ & $\bullet$ & 24.325 & $\bullet$ & $\bullet$ \\
\hline
\end{tabular}


Table 2. The critical pressure values $[\mathrm{kPa}]$ of dumb-bell samples, calculated with several models similarly to Table 1 . The effect of a different sample shape can be evaluated comparing the pre-conditioning frequencies $0 \mathrm{~Hz}$ and $0.2 \mathrm{~Hz}$. Unstable indicates that no fitting is achieved and $\bullet$ specifies that no aneurysm was predicted. For $0 \mathrm{~Hz}, 0.2 \mathrm{~Hz}$ and $0.8 \mathrm{~Hz}$ the stiffest (first column) and softest (second column) stress-strain response were taken into account. Conversely, an average curve was chosen for $1 \mathrm{~Hz}$.

\begin{tabular}{|c|c|c|c|c|c|c|c|}
\hline \multirow{3}{*}{$\begin{array}{c}\text { Strain } \\
\text { energy } \\
\text { function }\end{array}$} & \multicolumn{7}{|c|}{ Pre-conditioning frequency } \\
\hline & \multicolumn{2}{|c|}{$0 \mathrm{~Hz}$} & \multicolumn{2}{|c|}{$0.2 \mathrm{~Hz}$} & \multicolumn{2}{|c|}{$0.8 \mathrm{~Hz}$} & \multirow[t]{2}{*}{$1 \mathrm{~Hz}$} \\
\hline & Stiffest & Softest & Stiffest & Softest & Stiffest & Softest & \\
\hline \multicolumn{8}{|l|}{ Ogden } \\
\hline $1^{\text {st }}$ order & $\bullet$ & $\bullet$ & $\bullet$ & $\bullet$ & $\bullet$ & $\bullet$ & $\bullet$ \\
\hline Ogden & & & & & & & \\
\hline $2^{n d}$ order & $\bullet$ & $\bullet$ & 15.346 & 18.080 & unstable & $\bullet$ & 23.542 \\
\hline Ogden & & & & & & & \\
\hline $3^{r d}$ order & unstable & unstable & unstable & unstable & unstable & unstable & 23.676 \\
\hline Neo-Hooke & 28.167 & 17.445 & 21.925 & 23.998 & 17.812 & 24.249 & 28.250 \\
\hline Arruda-Boyce & 23.512 & unstable & unstable & 20.504 & unstable & 20.473 & unstable \\
\hline Yeoh & $\bullet$ & $\bullet$ & $\bullet$ & $\bullet$ & $\bullet$ & $\bullet$ & 22.472 \\
\hline
\end{tabular}


Table 3. Fitting parameters estimated by Abaqus for each strain-energy function plotted in Fig. 4 are reported. Ogden $3^{\text {rd }}$ order resulted unstable.

\begin{tabular}{ll}
\hline Strain-energy function & Fitting parameters \\
\hline Ogden $1^{\text {st }}$ order & $\mu=193613.5[\mathrm{~Pa}] ; \alpha=3 ;$ \\
Ogden $2^{\text {nd }}$ order & $\mu_{1}=43014.2[\mathrm{~Pa}] ; \alpha_{1}=4.1$ \\
& $\mu_{2}=209780.2[\mathrm{~Pa}] ; \alpha_{2}=1.4 ;$ \\
Neo-Hooke & $C_{10}=171436.6[\mathrm{~Pa}]$ \\
Arruda-Boyce & $\mu=259278.9[\mathrm{~Pa}] ; \mu_{0}=278386.105[\mathrm{~Pa}] ; \lambda_{m}=3$ \\
Yeoh & $C_{10}=122362.3[\mathrm{~Pa}] ; C_{20}=4331.1[\mathrm{~Pa}]$ \\
\hline
\end{tabular}




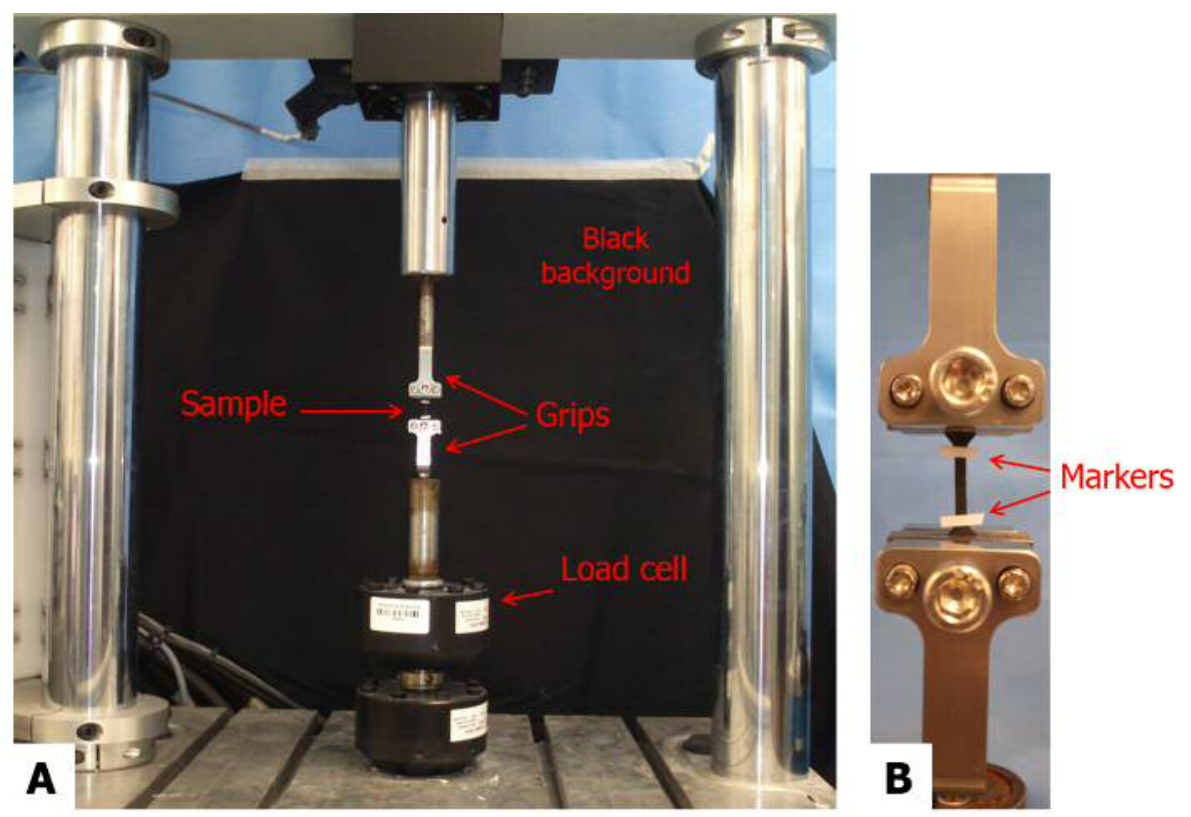

Fig. 1. A) Experimental setup adopted for the uniaxial tensile tests. The sample was kept in position by means of grips (B) and tested uniaxially up to failure. A video-extensometer was used to measure the displacement between the two white markers attached to the sample as detailed in $\mathrm{B}$. 

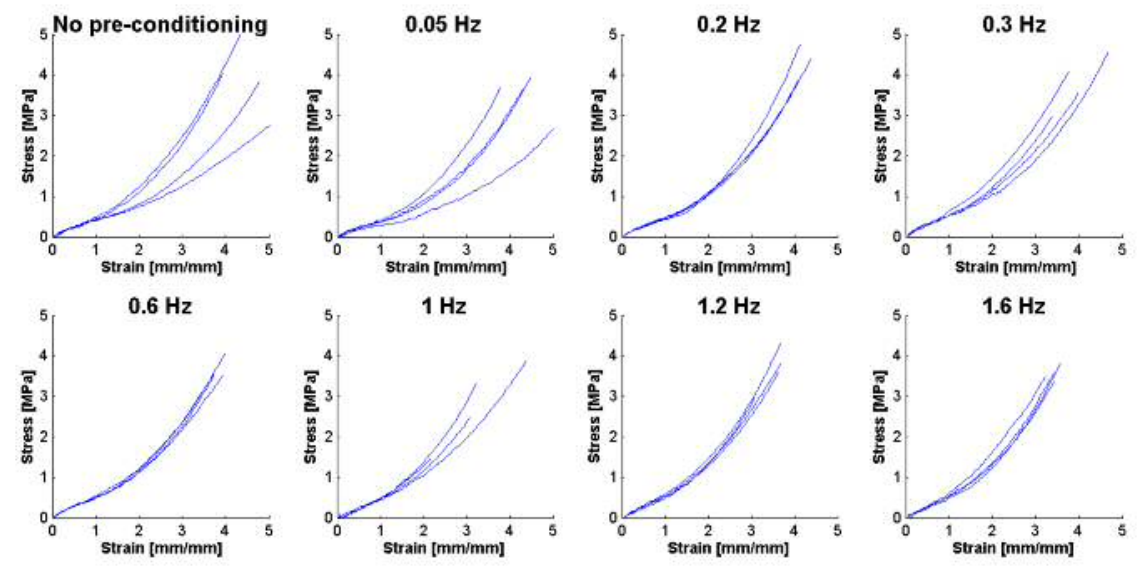

Fig. 2. Filtered uniaxial stress-strain response of rubber strips, pre-conditioned with 5 cycles at selected frequencies. Data obtained from no pre-conditioning, $0.2 \mathrm{~Hz}, 0.6 \mathrm{~Hz}$ and $1.6 \mathrm{~Hz}$ have been used in the FE model. 

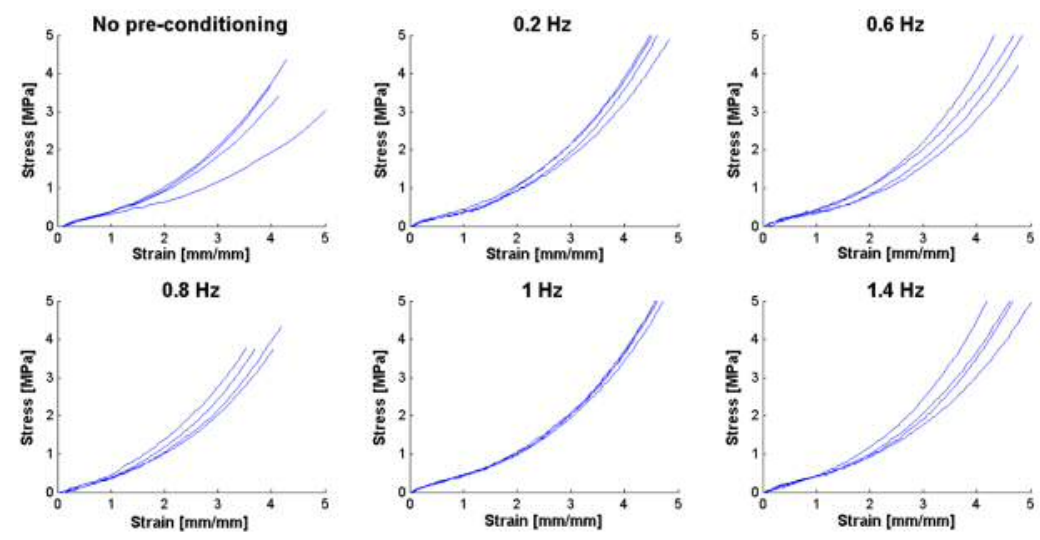

Fig. 3. Filtered uniaxial stress-strain response of rubber dumb-bells, pre-conditioned with 5 cycles at selected frequencies. Data obtained from no pre-conditioning, $0.2 \mathrm{~Hz}, 0.8 \mathrm{~Hz}$ and $1 \mathrm{~Hz}$ have been used in the FE model. 


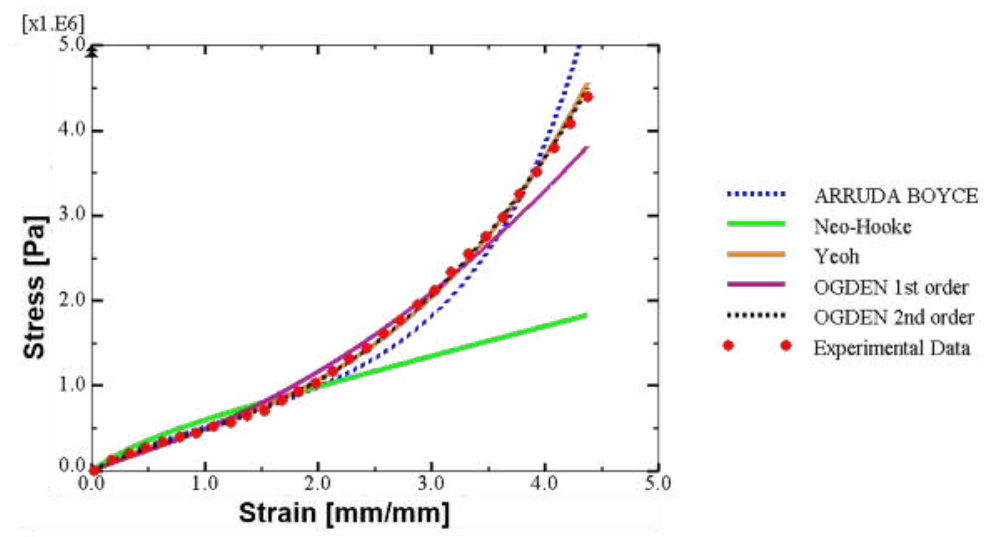

Fig. 4. Experimental data (red dots), collected from a strip pre-conditioned at $0.2 \mathrm{~Hz}$, fitted by different strain-energy functions in Abaqus. Fitting parameters are reported in Table 3. 

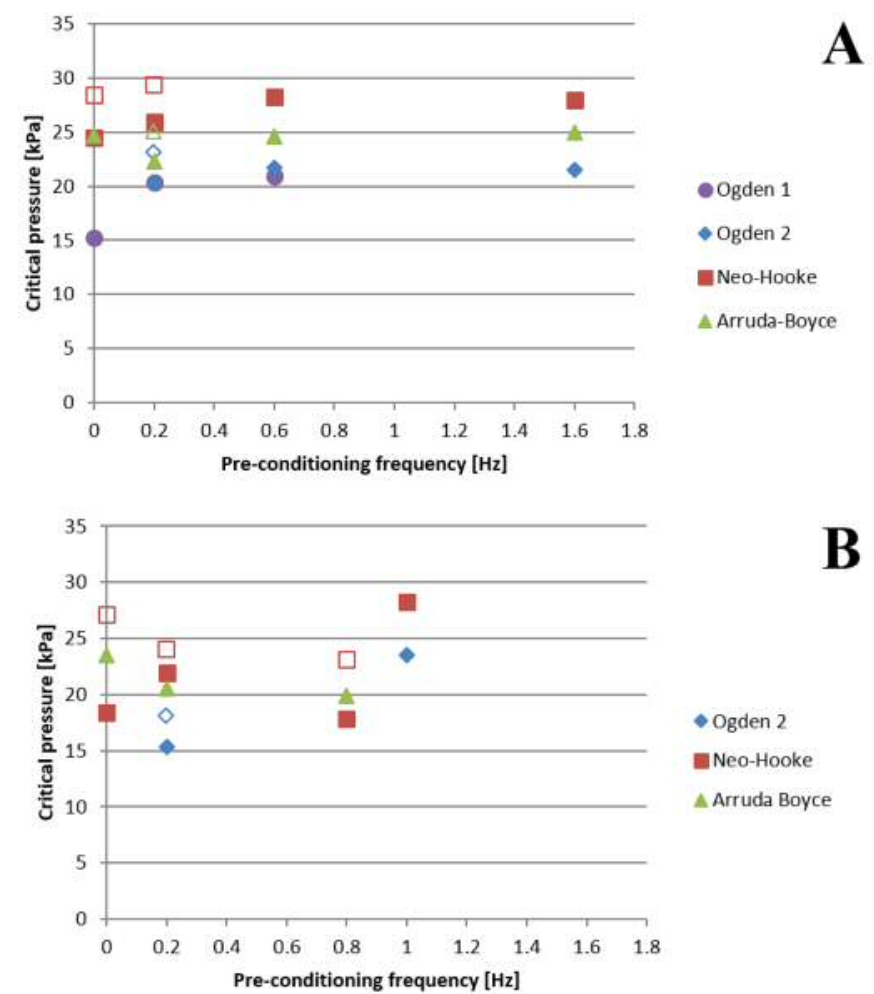

Fig. 5. Predicted critical pressures as a function of pre-conditioning frequencies: A) strips; B) dumb-bells. When two stress-strain curves, intended as the stiffest and the softest, have been selected, the higher values of critical pressure are indicated by open markers. 

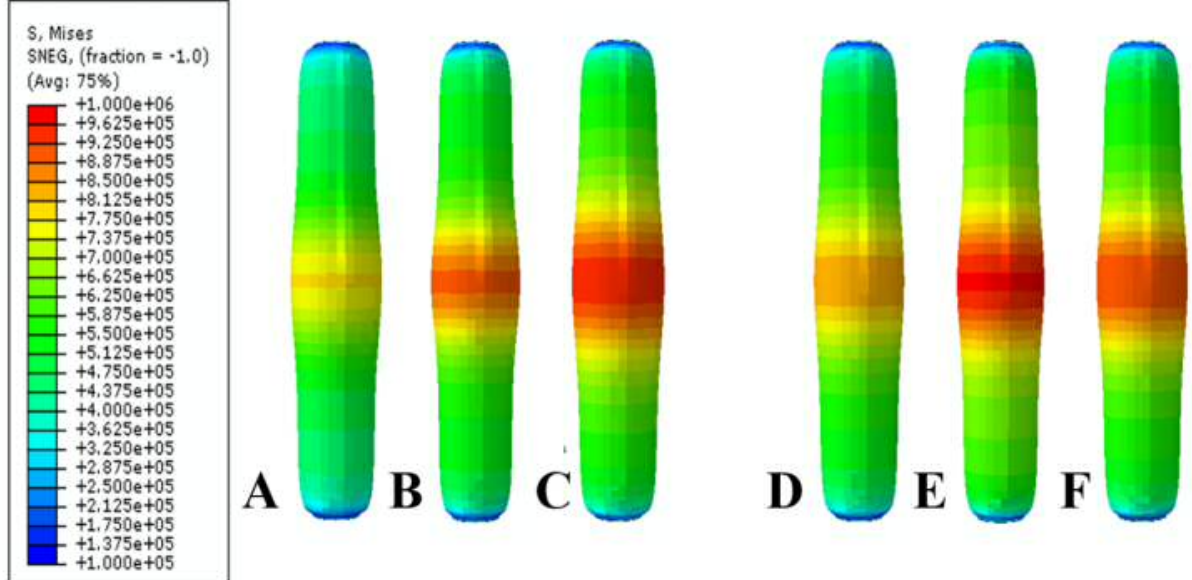

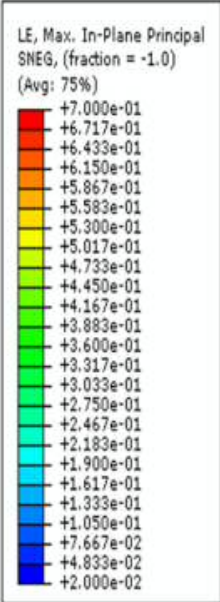
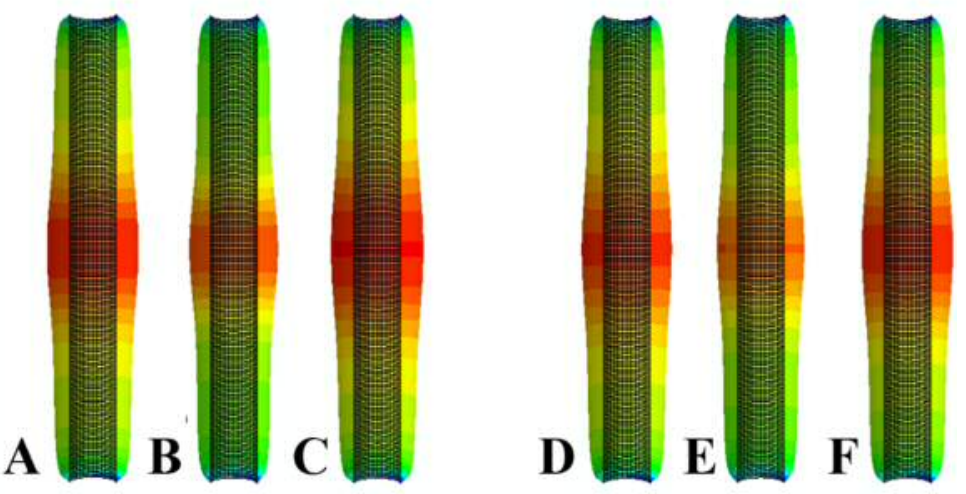

Fig. 6. Comparison of different aneurysm shapes predicted one step after the pressure reaches a critical point. The numerical results refers to experimental data for strips pre-conditioned at $0.2 \mathrm{~Hz}$, selecting two different stress-strain curves: the stiffest and the softest. The stiffest stressstrain response used: A) Ogden second order, B) Neo-hooke, C) Arruda-Boyce. The softest stressstrain response used: D) Ogden second order, E) Neo-hooke, F) Arruda-Boyce. The first row of results reports von Mises stress, whereas the second row shows the logarithmic strain valupes superimposed to the undeformed configuration. 
Table 1. Critical pressure values $[\mathrm{kPa}]$ calculated with the specified strain energy functions. The experimental data were obtained after strip pre-conditioning performed at the showed frequencies. Unstable indicates that no fitting is achieved and $\bullet$ specifies that no aneurysm was predicted. For $0 \mathrm{~Hz}$ and $0.2 \mathrm{~Hz}$ the stiffest (first column) and softest (second column) stress-strain response were taken into account. Conversely, an average curve was chosen for $0.6 \mathrm{~Hz}$ and $1.6 \mathrm{~Hz}$.

\begin{tabular}{|c|c|c|c|c|c|c|}
\hline \multirow{3}{*}{$\begin{array}{c}\text { Strain } \\
\text { energy } \\
\text { function }\end{array}$} & \multicolumn{6}{|c|}{ Pre-conditioning frequency } \\
\hline & \multicolumn{2}{|c|}{$0 \mathrm{~Hz}$} & \multicolumn{2}{|c|}{$0.2 \mathrm{~Hz}$} & \multirow[t]{2}{*}{$0.6 \mathrm{~Hz}$} & \multirow[t]{2}{*}{$1.6 \mathrm{~Hz}$} \\
\hline & Stiffest & Softest & Stiffest & Softest & & \\
\hline \multicolumn{7}{|l|}{ Ogden } \\
\hline $1^{\text {st }}$ order & 15.275 & $\bullet$ & $\bullet$ & 20.316 & 20.892 & $\bullet$ \\
\hline Ogden & & & & & & \\
\hline $2^{n d}$ order & unstable & $\bullet$ & 20.215 & 23.111 & 21.734 & 21.489 \\
\hline Ogden & & & & & & \\
\hline $3^{r d}$ order & unstable & - & unstable & unstable & unstable & unstable \\
\hline Neo-Hooke & 24.447 & 28.392 & 25.931 & 29.327 & 28.290 & 27.951 \\
\hline Arruda-Boyce & unstable & 24.640 & 22.233 & 25.014 & 24.567 & 25.009 \\
\hline Yeoh & 17.358 & $\bullet$ & $\bullet$ & 24.325 & $\bullet$ & $\bullet$ \\
\hline
\end{tabular}


Table 2. The critical pressure values $[\mathrm{kPa}]$ of dumb-bell samples, calculated with several models similarly to Table 1 . The effect of a different sample shape can be evaluated comparing the pre-conditioning frequencies $0 \mathrm{~Hz}$ and $0.2 \mathrm{~Hz}$. Unstable indicates that no fitting is achieved and $\bullet$ specifies that no aneurysm was predicted. For $0 \mathrm{~Hz}, 0.2 \mathrm{~Hz}$ and $0.8 \mathrm{~Hz}$ the stiffest (first column) and softest (second column) stress-strain response were taken into account. Conversely, an average curve was chosen for $1 \mathrm{~Hz}$.

\begin{tabular}{|c|c|c|c|c|c|c|c|}
\hline \multirow{3}{*}{$\begin{array}{c}\text { Strain } \\
\text { energy } \\
\text { function }\end{array}$} & \multicolumn{7}{|c|}{ Pre-conditioning frequency } \\
\hline & \multicolumn{2}{|c|}{$0 \mathrm{~Hz}$} & \multicolumn{2}{|c|}{$0.2 \mathrm{~Hz}$} & \multicolumn{2}{|c|}{$0.8 \mathrm{~Hz}$} & \multirow[t]{2}{*}{$1 \mathrm{~Hz}$} \\
\hline & Stiffest & Softest & Stiffest & Softest & Stiffest & Softest & \\
\hline \multicolumn{8}{|l|}{ Ogden } \\
\hline $1^{s t}$ order & $\bullet$ & $\bullet$ & $\bullet$ & $\bullet$ & $\bullet$ & $\bullet$ & $\bullet$ \\
\hline Ogden & & & & & & & \\
\hline $2^{n d}$ order & $\bullet$ & $\bullet$ & 15.346 & 18.080 & unstable & $\bullet$ & 23.542 \\
\hline \multicolumn{8}{|l|}{ Ogden } \\
\hline $3^{r d}$ order & unstable & unstable & unstable & unstable & unstable & unstable & 23.676 \\
\hline Neo-Hooke & 28.167 & 17.445 & 21.925 & 23.998 & 17.812 & 24.249 & 28.250 \\
\hline Arruda-Boyce & 23.512 & unstable & unstable & 20.504 & unstable & 20.473 & unstable \\
\hline Yeoh & $\bullet$ & $\bullet$ & $\bullet$ & $\bullet$ & $\bullet$ & $\bullet$ & 22.472 \\
\hline
\end{tabular}


Table 3. Fitting parameters estimated by Abaqus for each strain-energy function plotted in Fig. 4 are reported. Ogden $3^{\text {rd }}$ order resulted unstable.

\begin{tabular}{ll}
\hline Strain-energy function & Fitting parameters \\
\hline Ogden $\quad 1^{\text {st }}$ order & $\mu=193613.5 ; \alpha=3 ;$ \\
Ogden $\quad 2^{\text {nd }}$ order & $\mu_{1}=43014.2 ; \alpha_{1}=4.1 ; \mu_{2}=209780.2 ; \alpha_{2}=1.4 ;$ \\
Neo-Hooke & $C_{10}=171436.6$ \\
Arruda-Boyce & $\mu=259278.9 ; \mu_{0}=278386.105 ; \lambda_{m}=3$ \\
Yeoh & $C_{10}=122362.3 ; C_{20}=4331.1 ; C_{30}=37.2$ \\
\hline
\end{tabular}

